\title{
Les activités pré-orthographiques : nature, validité et conceptions
}

Jean-Pierre Jaffré et Marie-France Morin

\section{(2) OpenEdition}

1 Journals

Édition électronique

URL : http://journals.openedition.org/pratiques/1238

DOI : $10.4000 /$ pratiques. 1238

ISSN : 2425-2042

Éditeur

Centre de recherche sur les médiations (CREM)

\section{Édition imprimée}

Date de publication : 15 décembre 2008

Pagination : 189-208

\section{Référence électronique}

Jean-Pierre Jaffré et Marie-France Morin, « Les activités pré-orthographiques : nature, validité et conceptions », Pratiques [En ligne], 139-140 | 2008, mis en ligne le 15 décembre 2008, consulté le 08 mars 2021. URL : http://journals.openedition.org/pratiques/1238 ; DOI : https://doi.org/10.4000/ pratiques. 1238

\section{(c) Tous droits réservés}




\title{
Les activités pré-orthographiques: nature, validité et conceptions
}

\author{
Jean-Pierre Jaffré \\ MoDyCo , CNRS - Paris-Nanterre \\ Marie-France Morin
}

Faculté d'éducation, Université de Sherbrooke, Shrebrooke, Québec

\section{Introduction}

L'usage scolaire a longtemps considéré que les enfants devaient attendre d'avoir 6 ans, voire 7 , pour apprendre à lire et à écrire ${ }^{(1)}$. Cette position, qui conditionne aujourd'hui encore certains débats sur l'école maternelle ${ }^{(2)}$, découle d'un postulat selon lequel les activités de lecture et d'écriture nécessitent un certain degré de maturité cognitive. Or cette position a été remise en question dès la fin des années 60 et tout au long des années 70 , par des études qui se sont intéressées à la façon dont les très jeunes enfants « entraient» dans l'écrit. Originaires pour la plupart des États-Unis, ces études sont à l'origine du concept d'emergent literacy - ou litéracie émergente ${ }^{(3)}$. Privilégiant les représentations enfantines, elles réfutent une approche globale de la litéracie ${ }^{(4)}$ ainsi qu'une référence trop exclusive aux usages adultes. C'est de cette approche qu'il sera ici question, avec un intérêt particulier pour la production graphique et des activités qui nous appellerons "pré-orthographiques » dans la mesure où elles ne se réduisent pas à la reproduction de la norme sociale.

Les premières études sur les activités pré-orthographiques se sont d'abord inté-

(1) Le texte de cet article suit les recommandations des Rectifications orthographiques publiées au Journal Officiel du 6 décembre 1990 (Documents administratifs, n 100). Ce qui explique par exemple que des mots tels que « maitrise », « paraitre » ou « naitre » ne comportent pas d'accent circonflexe, ou que « assoir» s'écrive sans «e ».

(2) La parution récente d'un ouvrage intitulé Faut-il fermer les écoles maternelles? - aux éditions Michalon sous le pseudonyme de Jean Dazay - a ainsi rallumé la querelle entre ceux qui considèrent que la maternelle devrait être un lieu d'apprentissage et non de jeu, et ceux qui pensent au contraire qu'elle est là pour que les enfants s'éveillent et se socialisent.

(3) Rappelons que la litéracie désigne l'ensemble des activités de lecture et d'écriture, de l'apprentissage aux usages sociaux. Sur cette question, on peut se reporter à Barré de Miniac et al., 2004.

(4) Dans les années 70, Kenneth Goodman définissait la lecture comme une « devinette psycholinguistique ». 
ressées aux orthographes « inventées ». Traduite de l'anglais « invented spelling » ${ }^{(5)}$, cette expression désigne les initiatives de jeunes enfants aux prises avec une orthographe, qui reflètent les aspects graphiques auxquels ils sont d'abord sensibles. La notion d'invention traduit en l'occurrence le fait que ces premières productions, si elles ne respectent pas la norme sociale, ne sont pas pour autant dépourvues de toute logique orthographique.

Une réflexion sur les activités pré-orthographiques trouve tout naturellement sa place dans un projet consacré à la linguistique populaire dans lequel il est question de «pratiques linguistiques profanes ». Dans un document préparatoire au présent numéro, Achard \& Paveau parlent d'ailleurs d'« interventions spontanées sur la langue, qualifiées de fautes par les grammairiens et puristes, mais qui constituent une véritable pratique linguistique profane implicite si l'on considère que la faute constitue un discours sur la langue». Or les activités pré-orthographiques donnent lieu à bien des pratiques «non-conventionnelles », et notamment aux fameuses fautes d'orthographe, que Ferreiro \& Teberosky (1982) qualifiaient d' « erreurs constructives » ${ }^{(6)}$.

D'une façon plus générale en effet, et contrairement à ce que croient ceux qui se présentent volontiers comme des défenseurs de la langue, la production linguistique, à l'oral comme à l'écrit, se nourrit de «fautes ». C'est ce qu'a montré, voici des décennies, le linguiste suisse H. Frei (1929 : 19) quand il écrivait : « [...] la faute, qui a passé jusqu'à présent pour un phénomène quasi-pathologique, sert à prévenir ou à réparer les déficits du langage correct ». Ce que confirme, plus près de nous, Gadet (1999 : 597) quand elle explique que « les écarts par rapport au standard sont à regarder non comme des fautes mais comme indices des points où le système présente des difficultés pour ses locuteurs ».

Pour toutes ces raisons, si la défense d'une langue et de son orthographe peut se manifester par la surveillance jalouse des normes linguistiques et le refus de tout écart, elle peut également partir du principe que ces normes sont changeantes, les erreurs devenant un indice de ce changement. Cette seconde position, tout aussi culturaliste que la précédente, a l'avantage d'être confortée par les faits. L'histoire de la langue française illustre en effet l'importance de lois analogiques, actives aujourd'hui encore - l'un des exemples les plus connus étant la forme « il résoud» $(<$ «ésoudre ») pour « il résout» et, dans un avenir proche, la possible adjonction d'une cédille à « merçi » ou « içi » (< « leçon », « reçu »).

\section{Les productions pré-orthographiques ; définitions}

Mis en situation d'écrire et bien que ne disposant pas de la totalité des outils linguistiques nécessaires pour le faire, les jeunes enfants peuvent néanmoins pro-

(5) Read (1971) utilise l'expression invented spellings. Par la suite (1986), il utilise indifféremment les expressions creative spelling, beginning spelling et invented spelling. Chomsky (1979) emploie également l'expression invented spelling. À noter qu'en anglais, le terme spelling a un sens plus restreint que le terme orthographe en français.

(6) Ferreiro \& Teberosky (1982 : 7) font à ce propos le commentaire suivant : « Quand les enfants commettent systématiquement la même erreur, l'emploi du terme erreur sert surtout à masquer notre propre ignorance. Ce qui apparaît chez les enfants comme un manque de connaissance est en fait la preuve d'une degré surprenant de connaissance sur la langue. La production d'erreurs systématiques prouve en tout cas que l'apprentissage du langage ne passe pas par des éléments isolés qui s'associeraient progressivement les uns aux autres mais dépend plutôt de la formation de systèmes dans lesquels la valeur des parties est constamment redéfinie en fonction de ce qui change dans le système tout entier. " 
duire des traces pertinentes que nous associons ici à des activités pré-orthographiques. C'est dans ce sens que l'on parle d'orthographes inventées ou de graphies inventées. Chomsky (1979) explique à ce sujet qu'entre 4 et 6 ans, les enfants qui ne savent pas encore lire mais connaissent les lettres de l'alphabet et peut-être aussi certains sons, commencent à écrire des mots et des messages personnels, inventant chemin faisant leurs propres graphies. Read (1986:38), quant à lui, soutient que les graphies produites par les jeunes enfants sont essentiellement créatives : «Beaucoup de ces graphies ne peuvent pas avoir été apprises des adultes ou recopiées dans les livres; elles doivent être construites à partir d'une connaissance du nom des lettres, d'une conception de l'orthographe standard et d'un sens aigu des sons qui sont à la base du langage parlé. » La notion d'invention ne désigne donc pas une création ex-nihilo mais bien une création « réfléchie » qui s'exerce dans le cadre d'une écriture donnée.

Des productions graphiques qui ne respectent pas la norme orthographique peuvent en effet présenter un certain degré de pertinence, et cela d'autant plus qu'elles sont le fait de très jeunes enfants. Ces productions, parce qu'elles n'ont pas pour objectif de reproduire des formes normées, sont à prendre comme un indice de la façon dont les enfants " pensent» une orthographe. Leur analyse permet surtout de décrire des modalités spécifiques de traitement cognitif et de les envisager selon divers points de vue. La notion de «production pré-orthographique » regroupe en effet des approches différentes. Certaines préfèrent mettre l'accent sur les spécificités de la démarche d'acquisition, et notamment sur la part d'invention impliquée dans les productions - les « orthographes inventées »; d'autres, tout en considérant la démarche active de l'apprenant, mais plus soucieuses de l'accompagnement d'autrui vers une norme orthographique, s'interrogent sur la pertinence de ces productions et sur leur éventuel effet facilitateur - les « orthographes approchées 》.

Les orthographes inventées - Historiquement premiers, les travaux sur les orthographes inventées n'avaient pas d'emblée un but éducatif ou didactique. Ils voulaient avant tout vérifier l'hypothèse selon laquelle la maitrise de l'orthographe ne débutait pas brutalement à 6 ou 7 ans avec l'apprentissage de la norme orthographique mais dépendait d'un parcours ontogénétique. Parce qu'ils ont vu le jour dans la sphère alphabétique, les travaux sur les orthographes inventées ont en outre privilégié les habiletés phonographiques à une époque où ces habiletés connaissaient un regain d'intérêt. Ils montraient notamment que les jeunes enfants apprenaient une part importante de l'orthographe non pas en reproduisant des exemples adultes ou en copiant les livres mais en associant à leur manière des lettres et les sons du langage oral. Si cet aspect de la création orthographique est nouveau pour l'acquisition, il l'est moins pour l'orthographe elle-même, spécialement en France. Confrontés à sa complexité, bien des usagers ont imaginé par le passé des formes plus accessibles. Ainsi, et pour ne citer que cet exemple, des femmes aristocrates du $18^{\mathrm{e}} \mathrm{s}$. utilisèrent une orthographe phonographique et transparente, connue sous le nom d'ortografe des dames (Goodman, 2002). Les travaux sur les peu-lettrés du $19^{\mathrm{e}} \mathrm{s}$. ont eux aussi mis l'accent sur l'intérêt d'une orthographe moins complexe (Branca-Rosoff \& Schneider, 1994).

Vers des orthographes approchées - Cette première série de travaux a donc montré que l'apprentissage de l'orthographe ne commençait pas par la reproduction de formes normées mais impliquait un lent travail d'appropriation - jusque là méconnu - au cours duquel s'exprimait une intelligence de l'écrit d'autant plus créative qu'elle portait sur des principes - en l'occurrence alphabétiques - plutôt que sur des faits particuliers - des mots. Ce constat posait du même coup des ques- 
tions nouvelles, plus nettement pédagogiques, qui avaient le souci d'évaluer la distance entre ces productions enfantines et la norme orthographique, et d'envisager la meilleure manière de les rapprocher - d'où des appellations telles que « orthographes approchées ». Une telle démarche implique toutefois le recours à des modèles complexes de l'apprentissage qui permettent de dire si des productions erronées préparent la maitrise orthographique. Il ne s'agit pas pour autant, contrairement à ce qui se dit parfois, de faire crédit aux enfants en louant de façon systématique la qualité de leurs productions. Celles-ci ne sont, dans le meilleur des cas, que l'indice de représentations sur lesquelles il faut agir pour qu'elles se rapprochent de productions plus conformes à la norme. Si l'évaluation s'en tenait à la surface des faits, elle devrait certes constater la non pertinence de telles productions et même s'interroger sur le risque d'installer des usages fautifs. Mais en fait les productions pré-orthographiques confirment que l'apprentissage de l'orthographe nécessite aussi - et même d'abord - une intelligence du fonctionnement de l'orthographe dont les faits de surface ne sont que le résultat.

Cette différence d'objectifs ne doit cependant pas faire oublier que toutes ces approches attachent une importance particulière à ce que font les enfants, et à ce qu'ils disent. En effet, nous considérons que les orthographes inventées et les orthographes approchées doivent être envisagées dans une vision complémentaire et interactive du processus d'acquisition de la langue écrite, qui met l'accent tantôt sur un volet psycholinguistique, tantôt sur un volet didactique. À cet égard, les productions pré-orthographiques - terme générique qui englobe les deux précédentes - relèvent d'un ensemble de pratiques qui considèrent que la transmission des connaissances n'est pas la panacée. Et même si elle a son importance, elle ne saurait constituer une fin en soi ; pour être efficace, elle doit composer avec les profils cognitifs d'enfants dont les productions pré-orthographiques témoignent.

\section{De la notion d'invention}

L'invention des enfants - Comment faut-il comprendre la notion d'invention dans orthographes inventées ? Considérer en effet que les productions pré-orthographiques relèvent d'une création ex nihilo aboutirait à un contresens préjudiciable aux productions pré-orthographiques elles-mêmes. Tout prouve au contraire que ces productions sont une réponse aux stimulus de l'environnement. C'est d'ailleurs pourquoi elles peuvent être comprises comme un indice des représentations enfantines, à un moment donné du développement. Cette conception impliquée de l'invention a d'ailleurs une portée plus large. L'action d'inventer implique en effet que la force de l'imagination créatrice soit mise au service de quelque chose de nouveau ${ }^{(7)}$. Ainsi comprise, l'invention est synonyme de créativité et peut se définir comme " un ensemble des comportements déroulés en réponse à un certain type de situation » (Rouquette, 1973 : 112).

Le concept d'invention doit prendre la mesure du contexte social dans lequel vivent les enfants, c'est-à-dire d'un environnement où le statut de l'écriture découle tout naturellement de la tradition écrite, et d'un ensemble de facteurs qui font de l'écriture un moyen d'action indispensable. Cette activité est en outre étroitement dépendante des compétences cognitives des sujets, ce que Changeux (1998: 58) décrit comme un « système nerveux central qui projette en permanence ses hypothèses sur le monde extérieur, les met à l'épreuve et donne du sens à ce qui

(7) Le Trésor de la Langue Française (tome 10, p. 516) définit inventer par « [...] trouver par la force de l'imagination créatrice » et « réaliser le premier quelque chose de nouveau». 
n'en a pas ». S'y ajoutent les arguments psychologiques de spécialistes qui décrivent la force créatrice des enfants et la nécessité dans laquelle ils se trouvent d'y recourir pour simplement apprendre. Ferreiro (1990) parle de l'enfant comme d'un « artiste créateur », Karmiloff-Smith (1992) le compare à un linguiste en herbe. Autant de métaphores qui nous incitent à regarder les jeunes apprentis non pas comme des êtres-éponges qui se contenteraient d'absorber les connaissances mais comme des constructeurs de savoir, des bâtisseurs de théories.

Invention et orthographes - Ce type d'analyse vaut tout autant pour l'histoire des écritures. Bien que les avis sur la question demeurent partagés, les écritures ont rarement résulté d'une création stricto sensu. Il s'agit plutôt d'une invention « relative » perfectionnée par des techniques déjà présentes dans d'autres communautés linguistiques. Toute invention doit par conséquent être replacée dans un cadre sociolinguistique plus large. Confrontés à des situations nouvelles, les hommes - un groupe d'hommes plus avisés que les autres ? - utilisent autrement d'anciens états de connaissance. En l'occurrence, la nouveauté pourrait n'être que la reconfiguration de compétences disponibles jusque là, mais sous une autre forme. Cette remarque vaut d'ailleurs pour les écritures princeps, celles qui semblent avoir été créées de toutes pièces, à Sumer, dans l'Égypte ou la Chine anciennes, par exemple. Elle vaut également pour celles qui ont été modelées sur d'autres, antérieures. C'est le cas de l'orthographe du français dont le principe alphabétique résulte d'un emprunt à un alphabet latin lui-même emprunté aux Grecs.

L'invention peut certes prendre des formes différentes mais, dans tous les cas, elle est bien réelle. Les toutes premières écritures ont tendance à s'inspirer de tracés et de formes issus d'un environnement déjà riche en symboles graphiques, qu'elles utilisent à des fins linguistiques. Le dessin stylisé des sommets d'une montagne désignera la montagne, voire celui qui habite derrière la montagne, c'est-à-dire l'étranger. Plus tardivement, et d'une manière qui apparait plus subtile, les clercs du Moyen Âge ont adapté l'alphabet latin à la phonologie du français, puisant dans environnement qui leur était familier. Ils ont eu cependant bien du mal à s'affranchir des modèles antérieurs, optant pour des bricolages plus ou moins heureux. En témoignent les digrammes du français - « au », « on », « eu », etc. - nés de l'association de lettres existantes plutôt que de l'invention des lettres nouvelles, comme d'autres ont su le faire - voir le «ć » des orthographes slaves ou le « ö » des orthographes scandinaves.

\section{Les précurseurs}

Même si leurs buts théoriques ont pu différer, les travaux pionniers de Chomsky (1971, 1975, 1979), de Read (1971, 1975) ou de Ferreiro \& Teberosky (1982) ont tous mis l'accent sur la part de pertinence des réponses proposées par les jeunes enfants confrontés à l'écriture, hors toute copie. Il en résulte un regard différent sur les erreurs qui ne sont plus le reflet d'une norme mais la mise en œuvre de représentations linguistiques telles qu'elles se manifestent à un moment du développement. De fait, les travaux sur les productions pré-orthographiques des enfants se sont surtout intéressés à ce qu'elles révélaient des processus d'acquisition à l'œuvre.

Les travaux de Carol Chomsky (1979) privilégient les relations entre lecture et production écrite, à une époque où la première précède la seconde : on apprend d'abord à lire et ensuite seulement à écrire. Or Chomsky montre que, chez la plupart des enfants, la capacité d'écrire en représentant la forme phonique des mots, en totalité ou en partie, précède la capacité à lire. Mais au-delà de ces observations, son analyse prouve qu'en recourant à des lettres ou des blocs de lettres et à 
un traitement phonographique, les jeunes enfants peuvent s'essayer à écrire des mots avant d'en maitriser leur forme normée. C'est ce que Chomsky désigne par l'expression invented spelling, que nous avons traduite par " orthographes inventées ». Et si celles-ci diffèrent de l'orthographe standard, elles n'en sont pas moins systématiques et présentent de ce fait une certaine constance d'un enfant à l'autre. En écho à Chomsky, des travaux récents réaffirment la pertinence des activités pré-orthographiques en maternelle, sur le développement de la conscience phonologique (Vernon \& Ferreiro, 1999) ou sur le développement litéracique en contexte d'une scolarisation bilingue (Zecker, 2006). Ces chercheurs, et bien d'autres, considèrent en effet que les tentatives d'écriture s'avèrent un « produit » plus transparent que les activités de lecture pour saisir la manière avec laquelle l'enfant s'approprie la dimension phonologique de l'écrit.

Cette dimension était déjà omniprésente chez Charles Read, qui peut être considéré comme le promoteur majeur de la notion d'invention orthographique même s'il s'intéressait avant tout au niveau de conscience phonologique des enfants. Son bel ouvrage de 1986, publié sous le titre de Children's creative spelling, explique comment, plus que les adultes et que les enfants plus âgés, les jeunes enfants écrivent en notant les sons plutôt qu'en représentant des mots ou des morphèmes. Les graphies ainsi créées permettent d'analyser des procédures phonographiques et de répertorier les traits phonétiques auxquels ces enfants sont sensibles - Read est avant tout un linguiste. Si ces graphies peuvent parfois paraitre étranges, elles reposent en fait sur des principes tout à fait licites et notamment sur une tendance fondatrice des orthographes alphabétiques : représenter des sons identiques par des graphies identiques. Les graphies inventées éclairent également le développement d'enfants qui recourent à de critères concrets - les sons du langage - avant d'atteindre un niveau plus abstrait et de tenir compte d'autres types de relations.

Difficile de parler d'activités pré-orthographiques sans citer le nom d'Emilia Ferreiro. Mais à la différence de Read, qui aborde la question en linguiste, c'est en psychologue qu'elle propose une vision constructiviste de l'enfant qui écrit, dans la mouvance d'un Piaget dont elle fut l'élève. Ainsi, dans un ouvrage co-écrit en 1979 avec Ana Teberosky - mais cité ici dans sa traduction anglaise de 1982 - elle refuse de réduire l'apprentissage à un comportement passif. Elle considère au contraire que les enfants adoptent une attitude active à l'égard du langage qui les environne et qu'en essayant de le comprendre, ils formulent des hypothèses, ils recherchent des régularités et testent des prédictions. Ils construisent par conséquent une grammaire personnelle qui n'est pas simplement une copie déformée du modèle adulte mais une création originale. Au lieu de recevoir morceau par morceau une langue complètement fabriquée par les autres, les enfants reconstruisent la langue pour eux-mêmes et utilisant de façon sélective les informations que leur fournit l'environnement.

Cette approche montre comment le jeune enfant interagit avec l'écrit selon une séquence développementale complexe qui le conduit progressivement aux confins de la norme orthographique. Et le point fort des travaux de Ferreiro c'est son traitement de productions enfantines qu'elle n'évalue pas du point de vue de la norme orthographique mais de celui de l'enfant. Ainsi, au terme d'une analyse de l'évolution d'enfants hispanophones de 3-6 ans issus de milieux sociaux contrastés, elle conclut à la présence d'une psychogenèse ayant sa propre logique interne. Les informations en provenance du milieu sont alors incorporées dans des systèmes interprétatifs dont la succession n'est pas aléatoire, bien que la durée de chaque moment d'organisation - et, par conséquent, des âges d'apparition - dépende d'un ensemble d'influences diverses - sociales, familiales, éducatives, individuelles, etc. 
Un article tout entier ne suffirait pas à passer en revue tous les travaux sur les orthographes inventées ${ }^{(8)}$. Mais nous ne pouvons achever ce trop bref tour d'horizon sans faire état d'un ouvrage paru en 1980, sous le titre amusant de GNYS AT WRK - «genius at work ${ }^{(9)}$. Son auteure, Glenda Bissex, y décrit en détail les procédures graphiques choisies par son fils Paul alors âgé de 5 ans. Elle montre notamment comment, à travers l'exemple d'un mot tel que «direction » ${ }^{(10)}$, l'apprentissage privilégie un principe et une dynamique qui mènent progressivement à la graphie conventionnelle. Dans un texte plus tardif, Bissex (1984) revient sur ce processus d'apprentissage de l'écrit et décrit des enfants qui médiatisent les informations environnantes et les adaptent à la structure de leur esprit. Ainsi, chez Paul, l'apprentissage de l'orthographe, comme celui du langage en général, résulte d'expérimentations intensives, d'hypothèses qu'il teste, de l'incorporation d'informations nouvelles selon les processus d'assimilation et d'accommodation décrits par Piaget (1976).

De tous ces travaux émergent une constante essentielle : à l'oral comme à l'écrit, les enfants re-construisent leurs systèmes linguistiques. Cette re-construction ne passe cependant pas par l'accumulation de morceaux d'information mais par la découverte de principes et de règles de fonctionnement. En apprenant, les enfants font l'hypothèse d'un ordre et, de ce point de vue, on peut dire que l'esprit enfantin relève de structures historiques et universelles.

\section{Les activités pré-orthographiques en France et dans le monde}

Les premiers travaux sur les orthographes inventées ont d'abord porté sur l'orthographe de l'anglais et, avec ceux d'E. Ferreiro et ses collaborateurs, sur celle de l'espagnol. L'orthographe du français n'est cependant pas en reste, avec des approches qui, bien qu'employant des démarches différentes, ont abouti à des conclusions en grande partie similaires (Besse, 1990 ; Besse et al., 1993 ; Fijalkow, 1992 ; Fijalkow \& Fijalkow, 1991 ; Jaffré, 1992, 2000 ; etc.). Ces travaux ont confirmé que les enfants « entrent » très tôt dans l'orthographe mais qu'ils le font à leur manière. Ils produisent alors des calculs sous-jacents, avec un travail important d'appariement entre des unités graphiques - en l'occurrence des lettres de l'alphabet - et des unités phoniques - syllabes et phonèmes. Bien que la norme soit présente dans l'environnement social, sa maitrise implique un parcours cognitif qui permet, petit à petit, de produire des formes graphiques de plus en plus normées. La régularité des graphèmes est sur ce point décisive, comme le montre Morin (2007), tout spécialement pour une orthographe aussi complexe que celle du français.

Ainsi, et bien que les avis divergent sur la spécificité de ces phases et sur les modalités de leur coexistence, les travaux sur les orthographes inventées relevant de la sphère alphabétique s'accordent sur un point majeur : la compétence orthographique est d'abord une affaire d'habilité phonographique... ce qui n'est pourtant qu'une condition nécessaire. C'est néanmoins sur cette base que d'autres calculs vont voir le jour, qui relèvent cette fois des niveaux linguistiques dits supérieurs - lexique, morphologie notamment. C'est ce que l'on observe par exemple avec la fonction distinctive de l'accent espagnol dans jugo vs jugó ${ }^{(11)}$. Vaca Uribe (2007)

(8) Le chapitre 1 de Read (1986) peut utilement combler cette lacune.

(9) «Génie au travail», formule affichée par le fils de G. Bissex sur la porte de sa chambre.

(10) Écrit successivement DRAKTHENS $(5 ; 7)$, DRAKSHINS $(5 ; 8)$, DIREKSHONS $(7 ; 5)$, DIRECTOINS OU DIRECTIONS $(8 ; 1)$, DIRECTIONS $(8 ; 7)$.

(11) Ces deux mots espagnols, qui signifient respectivement «jus » et « je jouai », se distinguent par un accent, à l'oral comme à l'écrit. 
analyse l'évolution des procédures chez des enfants mexicains dont l'âge varie de 5 à 12 ans et il montre que si les enfants les plus jeunes ne tiennent pas compte de la différence (jugo dans les deux cas), au bout d'un certain temps néanmoins, un changement se produit. Les enfants proposent alors des réponses qui peuvent présenter une part d'originalité (jugo vs jugoo) avant d'être conventionnelles (jugo vs jugó).

Les études sur les activités pré-orthographiques des orthographes alphabétiques reprennent donc à leur compte les hypothèses initiées pour l'anglais. Mais désormais elles s'inscrivent dans un vaste courant international, en plein essor, et qui s'intéressent à des orthographes comme celles du japonais ou du chinois ${ }^{(12)}$.

Tsukada (2007) illustre ainsi les procédures qui conduisent de jeunes enfants japonais de maternelle et de $1^{\text {re }}$ année du primaire à la maitrise du syllabaire hiraga$n a$. La dimension créative des activités pré-orthographiques apparait dans l'exemple de «futari » («deux personnes »), qui s'écrit normalement à l'aide de deux caractères mais qu'un enfant peut écrire en se servant du chiffre « $2 »$ ([2ri]). En japonais « 2 » se lit en effet [futa]. Kato et al. (1999) s'interrogent quant à eux sur la nature des représentations graphiques d'enfants japonais âgés de 3 à 5 ans en leur faisant écrire la fameuse phrase d'E. Ferreiro - «papa frappe la balle» - qui, en japonais, devient «Otohsan-ga bohru-wo kerimasu » (papa, la balle, frappe). Les résultats confirment que les jeunes enfants procèdent d'abord en évoquant des images mentales auxquelles ils associent ce qui est écrit, puis considèrent que seuls sont écrits les acteurs et les actions et, finalement, prennent en compte les mots prononcés jusqu'à produire des segments phonographiques. Avec toutefois une spécificité notoire : en japonais, contrairement à ce qui se passe pour les écritures alphabétiques, la référence aux syllabes n'est pas transitoire.

Si le traitement des kana du japonais présente de nombreuses convergences avec celui des orthographes alphabétiques, il en va un peu différemment pour l'orthographe du chinois. Chi (1988) demande par exemple à de jeunes taïwanais âgés de 3 à 6 ans, qui parlent le mandarin, d'écrire leur prénom, et ce qu'ils souhaitent. Les productions vont du gribouillis à des formes normées, en passant surtout par catégories intermédiaires dont deux sont dominantes : des caractères homonymes $(40 \%)$ et des pseudo-caractères $(55 \%)$ souvent composés d'éléments proches de la norme. Les petits chinois de Taïwan seraient donc d'abord sensibles aux aspects visuographiques de leur orthographe et la dimension phonographique, quand elle existe, prendrait la forme des symboles transitoires qu'on leur enseigne au début de la $1{ }^{\text {re }}$ année - en l'occurrence bopomofo -, ou d'homonymes employés pour leur valeur sonore.

Mais ce point de vue ne fait pas l'unanimité comme le montrent les analyses de Shen \& Bear (2000). Ils considèrent en effet que les productions graphiques d'enfants chinois de la $1^{\text {re }}$ à la $6^{\mathrm{e}}$ années primaires confirment plutôt les conclusions développementales valant pour les orthographes alphabétiques, spécialement celle de l'anglais : d'abord phonologiques, ces erreurs deviennent orthographiques, puis sémantiques. D'autres études vont dans le même sens et plaident elles aussi pour un développement universel. Cela dit, si la dynamique est globalement la même pour toutes les orthographes, des différences n'en existeraient pas moins. En chinois par exemple, le nombre élevé de caractères augmente la quantité d'unités orthographiques, ce qui nécessite une bonne dose d'entrainement et de soutien, à l'école comme à la maison.

(12) Sur cette question, on peut se reporter à la synthèse coordonnée par Fijalkow (2007). 


\section{Ce que nous enseignent les activités pré-orthographiques}

Loin d'être les « culs-de-sac» décrits par le psychologue autrichien W. Dressler (1997), les savoirs « spontanés » dont témoignent les activités pré-orthographiques comportent donc une part de pertinence. Mais quelle est exactement leur validité en linguistique, psycholinguistique et didactique ? Sur ce point, les avis divergent quelque peu. D'une façon générale, les études dont on dispose, spécialement celles qui ont été publiées à la fin des années 80 et au début des années 90 , soulignent l'apport positif de ces activités, qu'elles contribuent à la découverte des relations spécifiques entre l'écrit et l'oral ou au développement des relations entre lecture et écriture - sur cette question, voir notamment Ehri (1989). Richgels (2001) va plus loin encore en affirmant que les changements intervenus au cours de ces trois dernières décennies dans le domaine de la production graphique des jeunes enfants sont une vraie révolution.

La dimension phonologique — Les études menées tout au long des années 70 ont souligné l'importance de l'encodage phonologique dans les orthographes inventées, spécialement lors des premières phases du développement orthographique. Une part importante de ces travaux a permis d'évaluer le niveau d'habilité phonologique en soulignant sa forte complémentarité avec l'orthographe. Read (1971), dont nous avons déjà souligné le rôle important en la matière, a ainsi démontré que de jeunes enfants ne sachant ni lire ni écrire étaient capables de porter une attention certaine aux phonèmes, pour aboutir à la sélection de lettres. Quelques années plus tard, le même Read (1975) a mis l'accent sur la précision des analyses phonétiques d'enfants de niveau préscolaire et l'aide qu'ils pouvaient en tirer pour écrire. De fait, les activités pré-orthographiques ont fourni un authentique outil d'évaluation, comme en témoignent de nombreuses études (Adams, 1990 ; Morris \& Perney, 1984 ; Richgels, 1986 ; Mann et al. 1987 ; etc.). Elles font par conséquent légitimement partie des batteries utilisées lors des débuts de la litéracie (voir Rieben et al., 2005).

Les premiers travaux sur les orthographes inventées sont pour l'essentiel le fait de linguistes et de psycholinguistes occupés à observer et décrire les processus graphiques. C'est ce qui explique que leurs résultats convergent vers un constat plutôt positif : les jeunes enfants sont très tôt capables de saisir des traits linguistiques dont la pertinence se situe à d'autres niveaux que ceux de la norme orthographique. Ils font du même coup la preuve qu'il existe bel et bien un apprentissage précoce de l'orthographe.

La notion d'orthographe inventée a mis du temps à se diffuser, notamment en France. Elle a pu susciter des sourires, dans le meilleur des cas, et parfois des critiques acerbes. En acceptant que les enfants commettent des erreurs, de telles activités ne les menaient-elles pas dans une impasse ? Pour intéressants qu'ils soient, les enseignements tirés de ces recherches ne constituent en effet qu'une première étape qui a pu apparaitre trop impressive, tant pour les pédagogues que pour les psycholinguistes. C'est ce qui explique qu'à la fin des années 90, apparaissent de nouvelles approches qui souhaitent évaluer la portée des activités pré-orthographiques selon un point de vue plus expérimentaliste. Sans que l'intérêt global de ces activités soit remis en question, des réserves sont alors faites sur leur efficience phonologique et surtout orthographique. Les meilleurs résultats seraient en fait obtenus par les enfants disposant d'un feedback normé, ce qui tendrait à prouver que les activités créatives ne doivent pas être séparées de la norme sociale (Rieben et al., 2005).

On peut s'interroger sur la portée de telles conclusions qui, pour des raisons de 
méthode, font trop souvent de la production graphique un exercice technique, une écriture de mots hors tout contexte. Pratiquées dans des ateliers, au service d'échanges linguistiques pédagogiquement encadrés, les activités pré-orthographiques coexistent en permanence avec la norme orthographique sans laquelle les enfants procèderaient en aveugle. Il n'en reste pas moins que leur objectif n'est pas de développer une connaissance orthographique normée mais de jouer un rôle décisif dans la mise en place d'une dynamique cognitive... qui se nourrit d'une confrontation à la norme. Leur efficacité passe par la mise en place d'une compétence phonographique de base, phase propédeutique indispensable de la litéracie. Richgels (1995) plaide lui aussi pour un tel enseignement, en contexte. Dahl et al. (1999) vont dans le même sens en montrant qu'un enseignement contextualisé aide à la mise en place d'une conscience phonologique quand bien même les enfants sont issus d'un milieu pauvre en références alphabétiques - le fameux effet Matthew de Stanovich (1986). Ce constat a d'ailleurs été récemment confirmé par une étude québécoise indiquant que la pratique régulière d'orthographes approchées en maternelle aide des élèves à risque à mieux apprendre l'écrit (Morin \& Montésinos-Gelet, 2007).

À y regarder de plus près, les processus en œuvre lors des activités pré-orthographiques ne sont d'ailleurs pas si éloignés de ceux qui sous-tendent la maitrise de l'orthographe conventionnelle. Certes, des notions comme celles de systématicité et d'abstraction ne suivent pas les mêmes règles que pour l'orthographe conventionnelle, et ne sont donc pas systématiques dans le même sens. Mais cette compétence est suffisamment importante pour que les enseignants s'en servent pour assoir des compétences plus larges. C'est ce qui explique que de nombreux chercheurs - Beers \& Henderson (1977), Gentry (1978), Paul (1976), Zutell (1977), etc. - aient intégré les activités pré-orthographiques dans des modèles développementaux plus larges.

Ces activités ouvrent en outre une fenêtre sur le fonctionnement cognitif des jeunes scripteurs. Leurs erreurs, surtout quand elles sont récurrentes, nous informent sur la façon dont le cerveau enfantin perçoit et analyse l'information graphique. On pourrait croire en effet que les enfants apprennent par imprégnation, à force de confrontations avec le modèle fourni par la norme orthographique. L'adulte enseignerait et l'enfant apprendrait. Mais l'observation de scripteurs débutants montre qu'une telle conception est très réductrice. De fait, à la lumière des activités pré-orthographiques, l'apprentissage apparait plutôt comme le résultat d'un double processus. L'un permet à l'apprenti de s'inspirer des formes disponibles dans l'environnement social et, à ce titre, l'observation effective des formes graphiques - et normées - est indispensable pour apprendre. S'en tenir à ce seul schéma équivaudrait toutefois à ignorer la part qui revient aux apprentis. De fait, les enfants ne voient et ne comprennent que ce que leurs représentations mentales leur permettent de voir et de comprendre. C'est la raison pour laquelle leurs productions écrites peuvent parfois paraitre surprenantes et, en tout cas, peu conformes aux attentes des adultes, d'autant plus satisfaites que les formes produites sont proches des formes sociales.

En fait, tous les enfants ne sont pas d'emblée sensibles aux principes de base d'une écriture. Pour certains d'entre eux, les lettres ne sont que des traces non fonctionnelles. C'est le cas d'Elsa (GS) ${ }^{(13)}$ quand elle écrit rapidement et sans commentaires la séquence graphique CEOLRBPAA qu'elle relit : «Je pars en voyage». D'autres en revanche se montrent capables de sélectionner les lettres selon des critères

(13) Les exemples sont extraits d'ateliers conduits en grande section de maternelle au cours des années 90 . 
plus fonctionnels. La séquence graphique EAOOUEAI, produite par Jean-Christophe (GS), reflète de façon très précise les syllabes orales du message suivant : « Eh l'oiseau on pourrait être ami ». Il trace d'abord E ( eh »), puis enchaine avec AO («l'oiseau »), OU (« on pourrait»), E (« être ») et termine sur AI (« ami »).

Si l'option d'Elsa témoigne d'un usage quelque peu gratuit des lettres, celle de Jean-Christophe est nettement plus motivée. Ces deux exemples illustrent en tout cas la spécificité des univers mentaux enfantins; ils sont aussi révélateurs de l'avancée décisive que constituent les associations entre la forme phonique des mots et leur forme graphique. Parce qu'elle s'appuie sur la valeur phonique contenue dans la dénomination des lettres, telle au moins que la définissent les pratiques sociales, l'épellation est dans ce domaine un excellent déclencheur d'habilités phonographiques. Ainsi, les lettres-voyelles du français («a», «i», «u»...) se prêtent mieux à cet exercice que les lettres-consonnes. Le nom des premières correspond en effet à la fonction phonographique tandis que celui des secondes coïncide seulement avec une rime - «c », «m», «1»... (Jaffré, 1992). Mais toutes les écritures n'offrent pas sur ce point les mêmes possibilités (Treiman, 2004). Quoi qu'il en soit, ce travail d'appariement, s'il est typique des orthographes alphabétiques, est également possible en Chine ou au Japon, sous des formes voisines à celles que l'on trouve en Europe. S'y ajoute cependant une autre dimension, due à la présence de caractères - hanzis, kanjis - qui présentent des récurrences morphologiques et lexicales absentes des orthographes occidentales.

Ontogénèse et morphogenèse - Les activités pré-orthographiques livrent également, et de manière indirecte, des informations sur la genèse des orthographes, c'est-à-dire sur leur histoire et leur développement. Sans aller jusqu'à prétendre, comme le fit jadis Jean Piaget, que le développement enfantin - l'ontogenèse - récapitule celui des espèces - la phylogenèse -, de nombreux chercheurs ont admis que des convergences étaient possibles entre le cheminement des enfants au cours de leurs tout premiers apprentissages et le développement des orthographes elles-mêmes - leur morphogenèse. Dans le cadre de cet article, nous nous contenterons de faire état de l'émergence des processus phonographiques, qui a joué un rôle majeur dans l'histoire de l'écriture et dont on retrouve des manifestations dans les premières productions graphiques des enfants (pour un exposé détaillé, se reporter à Fayol \& Jaffré, 2008).

Les premières écritures ont d'abord eu tendance à céder à la tentation de l'idéographie. À leurs débuts, en Mésopotamie, dans l'Indus ou en Chine, pour ne citer que ces cas, les écritures ont en effet opté pour des signes-mots dont le tracé s'inspirait de la forme des objets ou des notions représentés. En sumérien par exemple, la notion de /tête/ (SAG) était rendue par le dessin stylisé d'une tête qui, associé à celui d'un bol, signifiait/manger/ (Cooper, 1996). Mais ces signes avaient bien du mal à représenter des notions plus abstraites, plus linguistiques. C'est ce qui explique que, renonçant à une représentation iconique et plus ou moins codifiée du monde, les écritures aient finalement sélectionné la forme sonore des langues qui servait déjà à médiatiser du monde. Parce qu'elles sont en nombre limité, les unités de la phonographie - syllabes ou phonèmes - sont en effet les garants d'une économie que la seule représentation des signes linguistiques ne peut assumer.

Or les jeunes enfants ont tendance à suivre un cheminement similaire. L'idée selon laquelle une lettre de l'alphabet représenterait un son leur est d'abord étrangère. Les lectures adultes les incitent plutôt à considérer que la trace graphique réfère à des significations, en réception comme production. Cette "idéographie » enfantine peut prendre des formes différentes : dessins, copie ou production de graphies - lettres ou pseudolettres - auxquelles sont associées des significations 
souvent labiles qui ne valent qu'au moment de la production (Bousquet et al., 1999). Aucune de ces traces graphiques ne résulte en tout cas d'un appariement entre une forme graphique et des sons du langage, qu'il s'agisse de syllabes ou de phonèmes. À cet égard, on peut dire que les productions d'Elsa et de Christophe (ci-dessus) se situent aux antipodes l'une de l'autre. Elles permettent toutefois de mieux comprendre en quoi l'ontogenèse graphique rappelle certaines options de l'histoire des écritures, la phonographie permettant une économie structurelle et la créativité linguistique là où échoue un système basé sur la représentation exclusive d'unités logographiques.

Bien des recherches ont établi des parallèles entre ces principes fondamentaux des systèmes d'écriture et les traces écrites par les jeunes enfants. Parmi divers exemples, dont le dernier en date est celui de Pelletier \& Lasenby $(2007)^{(14)}$, citons celui de Ferreiro (1994) ${ }^{(15)}$ qui rompt certes avec l'idée piagétienne selon laquelle l'ontogenèse récapitulerait la phylogenèse mais admet que l'histoire peut aider à mieux comprendre le développement enfantin. Les relations souvent complexes du dessin et de l'écriture renforcent elles aussi l'idée d'un saut qualitatif dans les productions graphiques des jeunes enfants. S'ils utilisent d'abord le dessin comme une forme privilégiée d'expression graphique, ils recourent ensuite à des tracés plus abstraits, et parfois à des lettres... dans un but iconique. C'est ainsi que certains enfants établissent une relation entre la taille du référent et celle de la représentation graphique qu'ils en donnent, l'écriture du mot « train » nécessitant donc plus de lettres que celle de « libellule » (Ferreiro \& Teberosky, 1979).

\section{Activités pré-orthographiques et apprentissage}

Les jeunes scripteurs recourent en fait à des procédures différentes. Ils utilisent parfois des mots qu'ils connaissent plus ou moins globalement - «papa », « maman », « bonjour », etc. -, ou bien recopient des informations environnantes (Rieben et al., 1989) ou encore créent des formes graphiques originales en recourant à des compétences plus systématiques. Pour l'apprentissage, ces procédures ne présentent pas le même intérêt. Les deux premières demeurent en effet des processus fermés qui limitent le potentiel productif des jeunes enfants. La troisième - que nous avons désignée par les expressions « orthographe inventée » ou « orthographe approchée »- présente au contraire une dimension dynamique qui alimente la production graphique et touche plus directement au cœur du système d'écriture. Elle incite les enfants à saisir les principes fondamentaux d'un objet de connaissance donné et, ce faisant, permet de faire face à des situations nouvelles. Ainsi comprise, la production graphique relève de critères propres à la résolution de problèmes : face à une situation nouvelle, il faut créer des réponses relativement originales, en s'appuyant sur des états de connaissance disponibles. Et indépendamment de toutes situations expérimentales, le contexte pédagogique doit permettre la mise en place de conditions favorables à l'expression d'une telle activité créatrice.

Dans cette perspective, l'étude de Morin \& Montésinos-Gelet (2004) a permis

(14) Dans cette étude longitudinale sur les productions de jeunes anglophones, Pelletier \& Lasenby décrivent la transition entre deux modalités graphiques : la première, proche de la pictographie, se caractérise par une représentation indifférenciée des nombres et des concepts tandis que la seconde, linguistique, les différencie. C'est à partir de ce constat que Pelletier \& Lasenby établissent une relation avec les Sumériens qui ont d'abord pratiqué une représentation syncrétique des nombres et des concepts avant de les distinguer.

(15) Cette contribution est issue de l'ouvrage de Keller-Cohen (1994), écho d'une confrontation interdisciplinaire entre psycholinguistes et historiens de l'écriture. 
de constater que des pratiques d'orthographes approchées en maternelle favorisait le développement d'habiletés stratégiques chez les jeunes scripteurs. Plus précisément, l'analyse des verbalisations de sujets fréquentant la maternelle québécoise (âge moyen : 6,3 mois) a montré que le groupe impliqué dans des activités d'orthographes approchées fréquentes tout au long de l'année verbalisait une plus grande variété de stratégies (phonologique, lexicale et analogique) qu'un groupe qui n'adoptait pas ce type de pratiques. Ce dernier - le groupe contrôle - a notamment délaissé la procédure lexicale en cours d'année pour développer la procédure phonologique. De tels résultats suggèrent que le travail des enfants sur la langue écrite encourage le développement de stratégies nécessaires à la maitrise du code écrit et ne se limitent pas à une stratégie phonologique, certes essentielle mais non suffisante

Activités pré-orthographiques et constructivisme - D'une façon générale, les études sur les activités pré-orthographiques ont contribué à mettre l'enfant au centre du dispositif éducatif. Non pas pour le glorifier, comme le prétendent les tenants d'un enseignement directif dont la seule référence est le modèle adulte, mais pour connaitre son point de vue afin de l'aider à optimiser ses performances. Cette option rappelle bien évidemment la notion de zone de développement proximal de Vygostski (1985). Elle désigne la distance entre ce que l'enfant peut effectuer ou apprendre seul et ce qu'il peut apprendre uniquement avec l'aide d'un tiers. Il semble en tout cas qu'une analyse des activités pré-orthographiques soit indissociable d'une théorie sous-jacente de l'apprentissage, inspirée à l'origine par les travaux de Piaget (voir les grandes lignes dans Piattelli-Palmarini, 1979) et, pour le domaine plus spécifique de l'écrit, reformulée et appliquée par Henderson et ses collaborateurs de l'École de Virginie (Henderson \& Beers, 1980), et bien entendu par Ferreiro (1984, 1985, 1987, 1990).

Le concept d'orthographe inventée ou approchée tel que nous le présentons ici relève donc d'une approche constructiviste de l'apprentissage, le milieu social exerçant un certain nombre de pressions qui contraignent l'individu à fournir des réponses plus ou moins adaptées. Cette interaction implique toutefois des états intermédiaires de connaissance pour lesquels la norme orthographique, même si elle n'est jamais perdue de vue, ne saurait constituer un outil d'évaluation adéquat. Ces phases intermédiaires impliquent en effet des réorganisations provisoires qui empêchent de concevoir l'acquisition en termes d'imitation ou de reproduction des faits environnants, mais dépendent de la saisie des principes fondamentaux capables de générer des faits récurrents. C'est d'ailleurs le caractère abstrait de cette connaissance qui autorise des anticipations. Car les apprentis scripteurs ne se contentent pas d'appréhender ce qui les environne pour le reproduire; ils appliquent à des domaines encore lacunaires des calculs plus généraux.

Nous avons déjà eu l'occasion de préciser que, chez les jeunes enfants, le traitement de l'information procédait d'une double dynamique avec, d'un côté, les informations délivrées par les adultes et le milieu social et, de l'autre, des représentations et des compétences qui différencient l'enfant de l'adulte (Gopnik \& Meltzoff, 1997). Ce sont bien évidemment ces aspects qui contraignent les productions pré-orthographiques. Pour les décrire, Siegler \& Jenkins (1989) recourent à la métaphore de l'atelier : l'esprit d'un enfant serait comme un atelier contenant une collection de matériaux - la connaissance - et d'objets - les processus d'apprentissage - qui servent à fabriquer de nouveaux produits - règles, stratégies, hypothèses, schémas, etc. L'atelier permet ainsi de répondre aux demandes qui lui sont faites, avec certaines réponses toutes prêtes et d'autres qui doivent être construites de toutes pièces. 
Une pluralité de procédures — La tendance première à mettre un accent particulièrement insistant sur la dimension phonographique des activités pré-orthographiques plaide finalement pour une démarche d'acquisition très linéaire : la phonographie d'abord, l'orthographe ensuite. Or des travaux plus tardifs sur la question font très souvent état d'une coexistence procédurale, la part d'invention telle que nous l'entendons ici pouvant s'appliquer à des domaines très différents (Varnhagen et al., 1997). Treiman (1993) constate ainsi que les débutants euxmêmes disposent d'une connaissance rudimentaire sur l'orthographe de l'anglais et s'en servent. Siegler (1995) décrit cette polyvalence procédurale à l'aide de la métaphore du chevauchement des vagues - overlapping waves. Ehri (1992) considère elle aussi que pour rendre compte du développement en orthographe, on ferait mieux de parler de séries de processus plutôt que de processus spécifiques. De la même façon, Templeton (1992) intègre plus qu'elle ne distingue les aspects phonologique, orthographique et morphologique de l'orthographe.

Les modèles optant pour des étapes statiques et qualitatives du développement sont donc de plus en plus concurrencés par des descriptions dynamiques qui associent plus étroitement connaissances et stratégies (Varnhagen et al., 1997; Treiman $\&$ Bourassa, 2000). En ce sens, des études récentes menées auprès de jeunes francophones (David, 2003 ; David \& Morin, 2008 ; Pasa, Creuset \& Fijalkow, 2006) ont contribué à appuyer cette conception du développement orthographique en adoptant une méthodologie qui analyse l'activité pré-orthographique à partir d'une analyse des écritures produites, mais qui tient également compte, et favorise, les commentaires métagraphiques des jeunes enfants. Globalement, ces études ont permis de rendre compte d'une variété de procédures en cohérence avec la complexité du système écrit du français (procédures logographiques, phonographiques, morphographiques) et ce, même en début d'apprentissage. Ces études ont également montré que les enfants de maternelle géraient la dimension phonologique du français à l'aide d'une variété de procédures qui pouvaient être mise en relation avec des facteurs d' ordre linguistique - tel le type de syllabe à produire (Pasa et al., 2006) -, ou encore d'ordre contextuel - tel les interventions pédagogiques (David \& Morin, 2008).

\section{Pour conclure : des activités pré-orthographiques à la didactique}

Les activités pré-orthographiques nous apprennent finalement que l'acquisition de l'orthographe - comme celle de bien d'autres domaines - ne concerne pas seulement l'école primaire mais débute bien avant 6 ans. Ce constat s'inscrit en faux contre le crédo des années 70 , qui a toujours ses défenseurs, selon lequel il faudrait attendre que les enfants soient équipés neurologiquement pour commencer à apprendre à lire et à écrire. C'est pour des raisons similaires que, dans les pays scandinaves, l'apprentissage de la lecture ne commence pas avant 7 ans (Godenir, 2001) ${ }^{(16)}$. De fait, comme ont pu le faire à une époque certaines publications sur l'apprentissage précoce de la lecture (Delogne, 1973 ; Cohen, 1982), les premiers travaux sur les activités pré-orthographiques ont révolutionné l'appro-

(16) Cette étude comparative des instructions officielles dans les pays de la communauté européenne précise que, si la plupart des pays envisagent l'apprentissage de la lecture à partir de l'âge de 6 ans, certains programmes se préoccupent de la lecture dès l'âge de 4 ans (Irlande, Irlande du Nord) ou de 5 ans (Angleterre, Pays de Galles, Écosse), alors que d'autres ne l'envisagent qu'avec l'enseignement obligatoire, c'est-à-dire à l'âge de 7 ans (Danemark, Suède, Finlande). 
che de l'écrit. Ils montraient en effet que, confrontés à l'écrit, les enfants peuvent fournir des réponses construites et non aléatoires, et sont sensibles à des phénomènes moins apparents mais aussi plus productifs que la norme orthographique. Et si les traces produites par ces enfants peuvent apparaitre déroutantes, c'est qu'on ne peut les interpréter en se référant à la seule norme orthographique.

La pratique des activités pré-orthographiques implique en outre des options didactiques qui se distinguent - sans nécessairement s'y opposer - de celles qui préconisent un diktat de la norme orthographique. Dans le premier cas, l'acquisition repose sur un cheminement progressif qui consiste à saisir les lois structurelles d'une orthographe afin de favoriser la maitrise progressive de la norme. Dans le second cas, la norme orthographique constitue une référence qu'il s'agit de reproduire, ce qui revient à considérer que l'acquisition est pour l'essentiel le résultat d'une re-production. Nous pensons quant à nous que l'orthographe, comme tous les objets de connaissance, doit faire l'objet d'appropriations progressives, à la mesure des habiletés cognitives des apprenants. Tout apprentissage suppose des balbutiements, des essais et des erreurs. Et de ce point de vue, les activités pré-orthographiques, comme d'ailleurs la plupart des «fautes» d'orthographe, nous informent sur la nature des représentations en œuvre et, le cas échéant, sur la meilleure manière de les transformer pour qu'elles se rapprochent de la compétence optimale. Comme le suggèrent David \& Morin (2008), cette façon de concevoir l'apprentissage de l'orthographe a certaines implications didactiques qui favorisent notamment l'observation de faits de langue, le questionnement collectif, la comparaison de formes erronées ou normées afin que les jeunes enfants puissent comprendre, de plus en plus finement, le fonctionnement de l'écrit.

La pratique des activités pré-orthographiques, et les options didactiques qu'elles privilégient, devraient finalement créer de nouvelles attentes chez les enseignants. Il ne s'agit plus en effet d'évaluer les productions enfantines à l'aune de la seule norme orthographique - même si celle-ci n'est jamais totalement perdue de vue - mais d'évaluer un degré de pertinence structurelle. Dans la sphère alphabétique, il peut notamment s'avérer important de contrôler la capacité d'un enfant à mettre en œuvre la «logique » alphabétique en évaluant la qualité de la représentation phonographique d'un mot par exemple. Lors d'un temps T de l'apprentissage, il peut en effet être plus rentable de savoir qu'en français, le son [i] a toute les chances de s'écrire avec la lettre « $\mathrm{i}$ » que de savoir écrire « correctement» deux ou trois mots. En pédagogie, il n'est cependant pas question d'exclure une option au profit exclusif d'une autre. La vision de la norme orthographique, sa fréquentation et sa pratique sont en effet tout aussi indispensables que les constructions alphabétiques. Les deux approches ont donc tout intérêt à coexister. De fait, le recours aux activités pré-orthographiques entend (re)donner à l'enfant une part d'initiative tout à fait indispensable. C'est dans la coopération bien comprise entre essais personnels et confrontation avec la norme sociale que l'on peut progressivement mettre en adéquation les réponses produites et les demandes d'une communauté linguistique. 


\section{Bibliographie}

ADAMS, M.J. (1990) : Beginning to read : thinking and learning about print, Cambridge, MA : MIT Press.

BARRÉ DE MiniaC, Ch., Brissaud, C. \& Rispail, M., éds. (2004) : La littéracie. Conceptions théoriques et pratiques d'enseignement de la lecture-écriture, Paris : L'Harmattan.

BEERS, J.W. \& HENDERSON, E.H. (1977) : « A study of developing orthographic concepts among first grade children ", Research in the Teaching of English, 11, 133-148.

BESSE, J.-M. (1990) : "L'enfant et la construction de la langue écrite », Revue française de Pédagogie, Paris : I.N.R.P., 17-22.

Besse, J.-M., DE GAulmYN, M.-M. \& Luis, M.-H. (1993) : « Du pouvoir lire son prénom au savoir lire-écrire », Études de Linguistique Appliquée, Paris : Didier Erudition, 91, 8-21.

BISSEX, G.L. (1980) : GNYS AT WRK : A Child Learn to Read and Write, Cambridge, MA : Harvard University Press.

— (1984) : « The child as teacher », in H. Goelman, A. Oberg \& F. Smith, éds., Awakening to literacy. Heinemann, 87-101.

Bousquet, S., Cogis, D., DuCARD, D., Massonnet, J. \& JAFFré, J.-P. (1999) : "Acquisition de l'orthographe et mondes cognitifs », Revue Française de Pédagogie, 126, 23-38.

BRANCA-ROSOFF, S. \& SCHNEIDER, N. (1994) : L'écriture des citoyens. Une analyse linguistique de l'écriture des peu-lettrés pendant la période révolutionnaire, Paris : Klincksieck.

ChangeuX, J.-P. \& RiCEuR, P. (1998) : La nature et la règle, Ce qui nous fait penser, Paris : O. Jacob.

CHI, M. M-Y. (1988) : «Invented spelling/writing in Chinese-speaking children : the developmental patterns ", Yearbook National Reading Conference, 37, 285-296.

CHOMSKY, C. (1970) : «Reading, writing, and phonology », Harvard Educational Review, XL, 287-309.

- (1975) : «Invented spelling in the open classroom », Word, special issue, 499-518.

— (1979) : «Approaching reading through invented spelling », in P.A. Weaver \& L.B. Resnick, éds., Theory and practice of early reading, vol. 2, Hillsdale, N.J. : Lawrence Erlbaum Ass., 43-66.

CoHEN, R. (1982) : Plaidoyer pour les apprentissages précoces, Paris : PUF.

CoOper, J.S. (1996) : «Sumerian and Akkadian », in Daniels, P.T. \& Bright, W., éds., The world's writing systems, Oxford University Press, section 3, 37-57.

DAHL, K.L., SCHARER, P.L., LAWSON, L.L. \& GROGAN, P.R. (1999) : «Phonics instruction and student achievement in whole-language first-grade classrooms », Reading Reasearch Quarterly, 34, 312-341.

DAVID, J. (2003) : «La dimension orthographique dans les écrits des jeunes enfants ", Les Dossiers des sciences de l'Éducation, 9, 29-39.

D AVID, J. \& MORIN, M.F. (2008) : «Écritures approchées : des procédures métagraphiques des jeunes apprentis-scripteurs aux pratiques d'apprentissage », dans J. Dolz \& S. Plane, éds., Formation des enseignants et enseignement de la lec- 
ture et de l'écriture. Recherches sur les pratiques - Actes du Symposium de Sherbrooke (Québec, Canada), REF 2007, 9-10 octobre 2007. Namur : Presses Universitaires de Namur, 19-41.

Delogne, R. (1973) : Apprendre à lire avant six ans, Éditions de l'Université de Bruxelles.

Dressler, W.U., éd. (1997) : Studies in Pre- and Protomorphology, Wien, Aut. : Verlag der Österreichischen Akademie der Wissenschaften.

EHRI, L.C. (1989) : «Apprendre à lire et à écrire les mots », dans L. Rieben \& C. Perfetti, dir., L'apprenti lecteur : Recherches empiriques et implications pédagogiques, Neuchâtel : Delachaux \& Niestlé, 103-128.

- (1992) : «Review and commentary : stages of spelling development», in S. Templeton \& D. Bear, éds., Development of orthographic knowledge and the foundations of literacy. Hillsdale, NJ : LEA, 307-332.

FAYOL, M. \& JAFFRÉ, J.-P. (2008) : Orthographier, Paris : Presses Universitaires de France.

FERREIRO, E. (1984) : « The underlying logic of literacy development », in H. Goelman et al., éds., Awakening to Literacy, Exeter, N.H. : Heinemann, 154-173. — (1988) : «L'écriture avant la lettre », dans H. Sinclair, dir., La production de notations chez le jeune enfant, Paris : PUF, 17-70.

— (1990) : «Literacy development : psychogenesis », in Y. Goodman, éd., How children construct literacy : piagetian perspectives, Newark, Del : IRA, 12-25.

- (1994) : « Two literacy histories : a possible dialogue between children and their ancestors ", in Keller-Cohen, D., éd., Literacy : interdisciplinary conversations. Creskill, NJ : Hampton Press, 115-128.

Ferreiro, E. \& TEBERosky, A. (1982) : Literacy before Schooling, Heinemann Ed. Books [traduction de Los Sistemas de Escritura en el Desarrollo del Niño, Mexico : Siglo XXI, 1979].

FiJALKow, J., éd. (1992) : «Y a-t-il une psychogenèse de l'écrit? », Dossiers de l'éducation, $\mathrm{n}^{\circ} 18$. Toulouse : PUM.

- (2007) : «Invented spelling in varied contexts », L1-Educational Studies in language and literature, 7, 3, 1-4.

FIJALKOW, J. \& FiJALKOW, É. (1991) : « L'écriture inventée ou cycle des apprentissages. Étude génétique », Les Dossiers de l'Éducation, 18, 5, 8, 125-167.

FREI, Henri (1929/1971) : La grammaire des fautes, Genève : Slatkine reprints.

GADET, F. (1999) : «La langue française au XX $\mathrm{X}^{\mathrm{e}}$ siècle », dans J. Chaurand, dir., Nouvelle histoire de la langue française, Paris : Seuil, 583-671.

GENTRY, J.R. (1977) : «A study of the orthographic strategies of beginning readers », Dissertation Abstracts International, 1979, 39 (07), 4017A (University Microfilms $\left.n^{\circ} 79-01-152\right)$.

GODENIR, A. (2001) : " La comparaison des programmes d'enseignement », in M.-C. Nyssen \& S. Terwagne (dir.), L'apprentissage de la lecture en Europe, Toulouse : Presses universitaires du Mirail

Goodman, K. (1982) : Language and Literacy : The Selected Writings of Kenneth S. Goodman, Routledge \& Kegan Paul.

Goodman, D. (2002) : "L'ortografe des dames: Gender and language in the Old Regime », French Historical Studies, 25, 2, 191-223.

Goptnik, A. \& Meltzoff, A.N. (1997) : Words, thoughts, and theories, Cambridge, Mass. : Cambridge University Press. 
Henderson, E.H. \& BeERS, J.W., éds. (1980) : Developmental and cognitive aspects oflearning to spell, Newark, Del. : International Reading Association.

JAFFRÉ, J.-P. (1992) : « Le traitement élémentaire de l'orthographe : les procédures graphiques », Langue Française, 95, 27-48, Paris : Larousse.

- (2000) : "Ce que nous apprennent les orthographes inventées », dans

C. Fabre-Cols, éd., Apprendre à lire des textes d'enfants, Bruxelles : DeBoeck/Duculot, 50-60.

KARMILOFF-S Mith, A. (1992) : Beyond modularity, A developmental perspective on cognitive science, Cambridge, Mass. : The MIT Press.

Kato, Y., UEDA, A., OZAKI, K. \& MuKaigaWA, Y. (1999) : « Japanese preschoolers' theories about the hiragana system of writing ", Linguistics and Education, 10, 2, 219-232.

KELlER-COHEN, D., éd. (1994) : Literacy : Interdisciplinary conversations, Cresskill, NJ : Hampton Press.

MAnN, V.A., TOBIN, P. \& Wilson, R. (1987) : « Measuring phonological awareness through the invented spelling of kindergarten children », Merill Palmer Quarterly, 33, 365-392.

MORIN, M.F. (2007) : "Linguistic factors and invented spelling in children : The case of learning writing in Canada », L1-Educational Studies in Language and Literature, 7(3), 173-189.

MORIN, M.-F. \& MONTÉSINOS-GELET, I. (2004) : «La diversité des procédures utilisées par le scripteur débutant en maternelle », Scientia Paedagogica Experimentalis, XLI (2), 301-325.

— (2007) : «Effet d'un programme d'orthographes approchées en maternelle sur les performances ultérieures en lecture et en écriture d'élèves à risque ", Revue des Sciences de l'Éducation, XXXIII (3), 663-683.

MORRIS, D. \& PERNEY, J. (1984) : « Developmental spelling as a predictor of firstgrade reading achievement », The Elementary School Journal, 84, 441-447.

PASA, L., CReuset, V. \& FiJAlKow, J. (2006) : «Écriture inventée : pluralité des traitements et variabilité selon la structure syllabique », Éducation et Francophonie, XXXIV (2), 85-103.

PAUL, R. (1976) : «Invented spelling in kindergarten », Young Children, 21 (3), 195-200.

Pelletier, J. \& L ASENBY, J. (2007) : « Early writing development in L1 Englishspeaking children », L1-Educational Studies in language and literature, 7, 3, 81-107.

Piaget, J. (1976) : Le langage et la pensée chez l'enfant, Neuchâtel : Delachaux \& Niestlé, $9^{\text {e }}$ édition.

Piattelli-Palmarini, M., éd. (1979) : Théories du langage. Théories de l'apprentissage. Le débat entre J. Piaget et N. Chomsky, Paris : Seuil.

READ, C. (1971) : «Pre-school children's knowledge of English phonology », Harvard Educational Review, 41, 1-34.

- (1975) : Children's categorization of speech sounds in English, Research Report $\mathrm{n}^{\circ} 17$, Urbana, IL, National Council of Teachers of English.

- (1986) : Children's Creative Spelling, Routledge \& Keagan Paul.

RICHGELS, D.J. (1987) : «Experimental reading with invented spelling (ERIS) : A preschool and kindergarten method», The Reading Teacher, 40, 6, 522-531.

— (1995) : «Invented spelling ability and printed word learning in kindergarten », Reading Research Quarterly, 30, 96-109. 
- (2001): Invented spelling, phonemic awareness, and reading and writing instruction, in Neuman \& Dickinson, éds., Handbook of Early Literacy research, The Guilford Press, 142-155.

Rieben, L., Meyer, A., \& Perregaux, C. (1989) : «Différences individuelles et représentations lexicales : Comment cinq enfants de six ans recherchent et copient des mots ", dans L. Rieben \& C. Perfetti, éds., L'apprenti lecteur : Recherches empiriques et implications pédagogiques. Neuchâtel : Delachaux \& Niestlé, 145-169.

Rieben, L., NTAMAKILIRO, L., GONTHIER, B. \& FAYOL, M. (2005) : « Effects of various early writing practices on reading and spelling », Scientific Studies of Reading, 9, 2, 145,166.

Rouquette, M.-L. (1973) : La créativité, Que sais-je? Paris : PUF, p. 112.

SHEN, H.H. \& BEAR, D.R. (2000) : «Development of orthographic skills in Chinese children », Reading and Writing, 13, 3-4, 197-236.

SIEGLER, R.S. (1995) : « How does change occur : a microgenetic study of number conservation », Cognitive Psychology, 28, 225-273.

SIEGLER, R.S. \& JENKINS, E. (1989) : How children discover new strategies, Hillsdale, NJ : Lawrence Erlbaum Ass.,

Silva, C. \& Alves MARTINS, M. (2003) : «Relations between children's invented spelling and the development of phonological awareness », Educational Psychology, 23, 1, 3-16.

STANOVICH, K.E. (1986) : « Matthew effects in reading : Some consequences of individual differences in the acquisition of literacy ", Reading Research Quarterly, 21, 4, 360-407.

TEMPleton, S. (1992) : « Theory, nature, and pedagogy of higher-order orthographic development in older students », in $\mathrm{S}$. Templeton \& D. Bear, éds., Development of orthographic knowledge and the foundations of literacy. Hillsdale, NJ : LEA, 253-278.

TREIMAN, R. (1993) : Beginning to spell : A study of first-grade children. New York : Oxford University Press.

- (2004) : « Knowledge about letters as a foundation for reading and spelling », in R.M. Joshi \& P.G. Aaron, éds., Handbook of Orthography and Literacy, Lawrence Erlbaum Associates, 581-599.

TREIMAN, R. \& BourassA, D. (2000) : « The development of spelling skill », Topics in Language Disorders, 20 (3), 1-18.

TSUKADA, Y. (2007) : "A study of invented spelling and developing orthographic concepts in Japanese ", L1-Educational Studies in language and literature, 7, 3, 5-29.

VACA URIBE, G. (2007) : «Invented Spanish spelling : stress and intonation », L1Educational Studies in language and literature, 7, 3, 109-123.

VARnhagen, C.K., MCCAllum, M. \& Burstow, M. (1997) : « Is children's spelling naturally stage-like ? ", in R. Treiman, éd., Reading and Writing, 9, 5-6, 451-481.

VERNON, S.A. \& FERREIRO, É. (1999) : «Writing development : A neglected variable in the consideration of phonological awareness », Harvard Educational Review, 69 (4), 395-415.

Vygotsky, L.S. (1985) : Pensée et Langage, Paris : Éditions Sociales, 269-270.

ZECKER, L. B. (2006) : «Learning to read and write in two languages : The deve- 
lopment of early biliteracy abilities », in C.A. Stone, E.R. Silliam, B.J. Ehren \& K. Appel, éds., Handbook of Language and Literacy: Development and Disorders. New York: The Guilford Press, 248-266.

ZuTELL, J.B. (1980) : " Children's spelling strategies and their cognitive development », in E.H. Henderson \& J. W. Beers, éds., Developmental and Cognitive Aspects of Learning to Spell. Newark, Del. : International Reading Association, 52-73.

\section{IFTRANGAS AUJOURDFUI}

Septembre 2008

N०162

Descriptions de la langue et enseignement

D. de Robillard : Langue(s)/systèmes/ didactiques, diversité, identités

F. Gadet et E. Guérin : Le couple oral/écrit dans une sociolinguistique à visée didactique

M.-A. Paveau : La langue sans classes de la grammaire scolaire

J. David: Variétés de la langue et diversité des approches grammaticales francophones

M.-M. Bertucci : Quelles descriptions de la langue parlée à l'école?

P. Cappeau : II manque des indéfinis!

B. Lavieu : Autour des compléments : grammaire scolaire et recherches linguistiques

C. Renvoisé : Donner sens à l'activité grammaticale

D. Manesse : Pour un enseignement de la grammaire minimal et suffisant

J.-L. Chiss : Postface : langue(s) et grammaire(s)

Revue de I'Association Française des Enseignants de Français

http://www.afef.org

Rédaction - Administration :Armand Colin, 21, rue du Montparnasse, 75006, PARIS.

Tél. 0144395121 - Courriel Rédaction : le-francais-aujourdhui@wanadoo.fr

\section{questions de communication}

\section{$n^{\circ} 5$ Qualifier des lieux de détention et de massacre}

sous la direction de B. Fleury et J. Walter

Histoire et mémoire du camp de la Neue Bremm

Parcours dans les camps de la seconde guerre mondiale

Des lieux de répression aux controverses mémorielles

\section{$\mathrm{n}^{\circ} \mathbf{6}$ Dynamique des peuples et constructions identitaires}

sous la direction de B. Fleury et J. Walter

Médiations des cultures en Lorraine

Médiations des cultures en Europe et au-delà.

PRIX AU NUMÉRO 20 euros (frais de port de 3,25 euros en sus)

ABONNEMENT ( 1 an, 2 numéros) 32 euros (frais de port de 6,50 euros en sus) Revue publiée avec le concours du Centre de recherche sur les médiations (Université Paul Verlaine-Metz) et le soutien du Conseil Régional de Lorraine et du Centre National du Livre et du CNRS

Presses universitaires de Nancy•pun@univ-nancy2.fr 